\title{
Controllable Four-Wave Mixing Based on Hybrid BEC-Optomechanical Systems
}

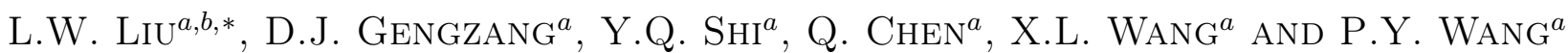 \\ ${ }^{a}$ College of Electrical Engineering, Northwest Minzu University, Lanzhou 730000, China \\ ${ }^{b}$ Key Laboratory for Electronic Materials of the State Ethnic Affairs Commission of PRC, \\ Northwest Minzu University, Lanzhou 730000, China
}

(Received February 18, 2019; revised version July 21, 2019; in final form July 24, 2019)

\begin{abstract}
We theoretically investigate the four-wave mixing (FWM) process based on hybrid optomechanical systems consisting of a cigar-shaped Bose-Einstein condensate (BEC), trapped inside an optical cavity with a moving endmirror. We can use a strong control field driving the cavity to control the bistable behavior of the steady-state photon number, the phonon number of the collective oscillation of the BEC and the phonon number of the mechanical resonator. Furthermore, we show how optomechanically induced transparency (OMIT) in the hybrid optomechanical systems can be used to control the four-wave mixing and enhance the intensity of the four-wave mixing. The calculated results show that the effect of the four-wave mixing can be controlled effectively by the pump strength, the frequency difference between the BEC and the moving end mirror, cavity decay rate, and effective coupling strength of the optical field with the moving mirror. Finally, the number peak of FWM can be controlled by modulating the frequency difference between the BEC and the moving end mirror and effective coupling strength of the optical field with the moving mirror.
\end{abstract}

DOI: 10.12693/APhysPolA.136.444

PACS/topics: Four-wave mixing; Bose-Einstein Condensate; Cavity Optomechanical

\section{Introduction}

Optomechanical systems have become a powerful platform for manipulating mechanical resonators and cavity fields $[1,2]$. There are many kinds of optomechanical systems, such us: two fixed mirrors [3], one fixed and another movable [4], a micro-mechanical membrane oscillating [5] and a Bose-Einstein condensate (BEC) inside two fixed cavity, and so on $[6,7]$. Recently, optical bistability has been extensively studied in the optomechanical systems [8-12]. On the one hand, the optical bistability can be obtained in the optomechanical system, where the cavity field is coupled to the mechanical resonator via the radiation pressure. On the other hand, in the presence of a strong control field, optical response of the optomechanical system to a weak probe field can be modified, leading to the phenomenon of optomechanically induced transparency (OMIT). Particularly, in resolved sideband regimes (the dissipation rate of the optical mode is smaller than the resonance frequency of the mechanical resonator), optomechanically induced transparency have been observed in various optomechanical systems [13-16]. Worldwide research in OMIT includes optical bistablility and four-wave mixing (FWM) [17], single photon quantum router [18], superluminal and ultraslow light propagation [19, 20], charge measurement, and so on [21-23].

As everyone knows, both in experiment and in theory the four-wave mixing has attracted considerable attention. In experiment, a lot of research has been

\footnotetext{
*corresponding author; e-mail: liuliw@xbmu.edu.cn
}

focussed on this aspect for a long time. There are reports of the enhancement of nondegenerate FWM based on EIT in a $\Lambda$-type three-level system of rubidium atoms [24]. In theory, there are reports of FWM based on the asymmetric quantum wells where OMIT has been observed [25] and a coupled Bose-Einstein condensate cavity system [26]. Herein, we show how hybrid optomechanical systems can be used for realization of the four-wave mixing by using OMIT.

In this paper a new kind of the four-wave mixing based on the hybrid optomechanical systems are designed, and the transmission properties are investigated numerically. Optomechanical systems with a cigar-shaped Bose-Einstein condensate trapped inside an optical cavity with a moving end-mirror have many advantages. Firstly, a strong coupling regime can be easily reached even with an ultralow pump power $[27,28]$. Furthermore, in the hybrid optomechanical systems, the intracavity laser field has a dual role: it excites a momentum side-mode of the condensate and acts as a nonlinear spring [29]. More importantly, the BEC can be trapped on a small scale and thus a robust miniature device can easily be implemented [30-32].

Motivated by these developments, we study the optical bistability and FWM in a hybrid optomechanical system in Sect. 2, where a cigar-shaped BEC is trapped inside an optical cavity with a moving end-mirror. We find the bistable behavior of the steady-state photon number, the phonon number of the collective oscillation of the BEC and the phonon number of the mechanical resonator. The numerical results in Sect. 3 show that the effect of the FWM can be controlled effectively by the pump strength, the frequency difference between 
the BEC and the moving end mirror, cavity decay rate, and effective coupling strength of the optical field with the moving mirror. We discuss the influence of thermal noise on the FWM in Sect. 4. A summary is presented in Sect. 5.

\section{The physical model}

The system considered in this paper is shown in Fig. 1, where a cigar shaped BEC of $N$ atoms of ${ }^{87} \mathrm{Rb}$ in its ground state is trapped in an optical ultrahigh-finesse Fabry-Pérot cavity. One mirror is free to move in the direction parallel to the cavity axis. In presence of radiation pressure from the cavity, the mirror moves harmonically with a frequency $\omega_{m}$ and is at equilibrium temperature $T[18]$. The cavity field is driven by a strong pump laser field accompanying a weak probe laser field along the cavity axis. The Hamiltonian of the optomechanical systems consisting of a cigar shaped BEC trapped inside an optical cavity with a moving end-mirror as

$$
\begin{aligned}
\hat{H} & =\hbar \omega_{c} \hat{c}^{\dagger} \hat{c}-\mathrm{i} \hbar E_{\mathrm{pu}}\left(\hat{c} \mathrm{e}^{\mathrm{i} \omega_{\mathrm{pu}} t}-\hat{c}^{\dagger} \mathrm{e}^{-\mathrm{i} \omega_{\mathrm{pu}} t}\right) \\
- & \mathrm{i} \hbar E_{\mathrm{pr}}\left(\hat{c} \mathrm{e}^{\mathrm{i} \omega_{\mathrm{pr}} t}-\hat{c}^{\dagger} \mathrm{e}^{-\mathrm{i} \omega_{\mathrm{pr}} t}\right) \\
& +\int_{0}^{L} \mathrm{~d} x \Psi^{\dagger}(x)\left[\frac{-\hbar^{2}}{2 m_{a}} \frac{\mathrm{d}^{2}}{\mathrm{~d} x^{2}}+V_{e x t}(x)\right] \Psi(x) \\
+ & \int_{0}^{L} \mathrm{~d} x \Psi^{\dagger}(x) \hbar U_{0} \cos ^{2}(k x) \hat{c}^{\dagger} \hat{c} \Psi(x) \\
+ & \frac{\hbar \omega_{m}}{2}\left(\hat{p}^{2}+\hat{q}^{2}\right)-\hbar G \hat{c}^{\dagger} \hat{c} \hat{q},
\end{aligned}
$$

where the first term describes the energy of the intracavity field, $\omega_{c}$ is the cavity frequency, $\hat{c}$ is the cavity field's annihilation operator. The second and third terms describe the energy of the strong pump laser field and the weak probe laser field. The classical light inputs with frequency $\omega_{\mathrm{pu}}$ and $\omega_{\mathrm{pr}}, E_{\mathrm{pu}}$, and $E_{\mathrm{pr}}$ are related to

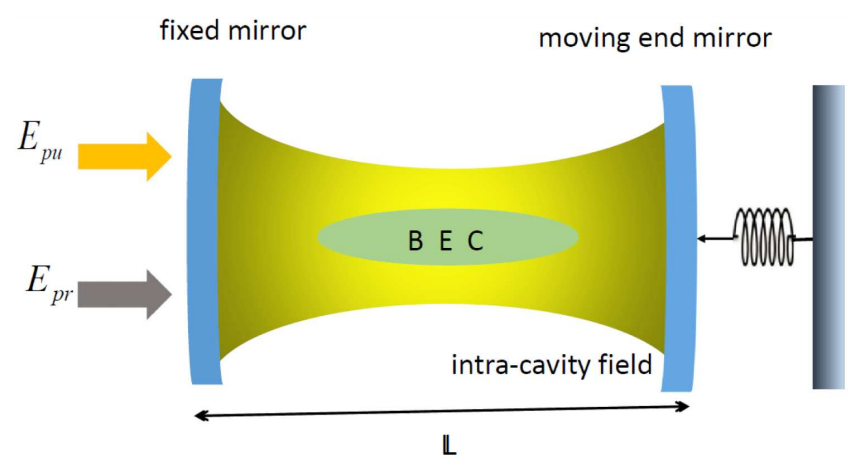

Fig. 1. The hybrid optomechanical system consisting of a Bose-Einstein condensate (BEC) trapped inside an optical cavity with a moving end-mirror. The cavity with decay rate $\kappa$ is driven through the fixed mirror by two lasers with frequency $\omega_{\mathrm{pu}}$ and $\omega_{\mathrm{pr}}$ and the end mirror is free to oscillate at mechanical frequency $\omega_{m}$. the laser power $P$, respectively, by $\left|E_{\mathrm{pu}}\right|=\sqrt{2 P_{\mathrm{pu}} \kappa / \hbar \omega_{\mathrm{pu}}}$ and $\left|E_{p r}\right|=\sqrt{2 P_{\mathrm{pr}} \kappa / \hbar \omega_{\mathrm{pr}}}$. The third and fourth lines describe the Hamiltonian of the condensate of the BEC in the case of weak atom-atom interactions and a shallow external trapping potential. Here, $\Psi(x)$ is a bosonic field annihilation operator, $m_{a}$ is the atomic mass, $V_{\text {ext }}$ is the external potential. The fifth term in fourth line describes the energy of the atom-cavity coupling interaction. Here, $U_{0}=g_{0}^{2} / \Delta_{a}$ is the maximum light shift which an atom experiences in the cavity mode, $\Delta_{a}=\omega_{\mathrm{pu}}-\omega_{c}$ is the detuning between the pump laser frequency and the atomic transition frequency, $g_{0}$ is the maximum coupling strength between a single atom and a single intracavity photon, and $k$ is the wave number of the light field. In our paper, we consider the strong coupling regime, $\left(N g_{0}^{2} /\left|\Delta_{a}\right| \gg \kappa\right.$, where $\kappa$ is the decay rate of the cavity and $N$ is the average number of atoms). The last line describes the Hamiltonian of the moving mirror and the mirror-cavity interaction, $\omega_{m}$ is the free mechanical frequency, $\hat{q}(\hat{p})$ is the position (momentum) operator of the mirror, while $G=\left(\omega_{c} / L\right) \sqrt{\hbar / m \omega_{m}}$ is the mirrorcavity coupling rate.

One can assumed, in the simple situation, that optical field is weak enough that only the first two symmetric momentum side modes with moment $\pm 2 \hbar k$ are excited by fluctuations due to atom-light interaction. For this purpose the discrete mode approximation is used. Moreover, only the first two symmetric momentum side modes with moment $\pm 2 \hbar k$ are excited by fluctuations, as a result of the atom-light interaction. Considering the parity conservation and the Bogoliubov approximation, the atomic field operator can be expanded to

$$
\Psi(x)=\sqrt{\frac{1}{L}} \hat{a}_{0}+\sqrt{\frac{2}{L}} \cos (2 k x) \hat{a}_{2},
$$

where $\hat{a}_{0}$ and $\hat{a}_{2}$ are bosonic annihilation operators for atoms in the zero-momentum state and side-mode components, respectively. The total number of atoms is $N=\hat{a}_{0}^{\dagger} \hat{a}_{0}+\hat{a}_{2}^{\dagger} \hat{a}_{2}$. As the population in the zeromomentum state is much larger than the population in the side-mode state, we can neglect the population in the side-mode state. We use $\hat{a}_{0}^{\dagger} \hat{a}_{0} \simeq N$, and $\hat{a}$ instead of $\hat{a}_{2}$. In a rotating frame at a driving field frequency $\omega_{p u}$, the Hamiltonian of the hybrid optomechanical systems is [29]

$$
\begin{aligned}
\hat{H} & =-\mathrm{i} \hbar E_{\mathrm{pu}}\left(\hat{c}-\hat{c}^{\dagger}\right)-\mathrm{i} \hbar E_{\mathrm{pr}}\left(\hat{c} \mathrm{e}^{\mathrm{i} \delta t}-\hat{c}^{\dagger} \mathrm{e}^{-\mathrm{i} \delta t}\right) \\
& +\hbar \Delta_{c} \hat{c}^{\dagger} \hat{c}+\hbar \omega_{m}^{\prime} \hat{a}^{\dagger} \hat{a}+\hbar g\left(\hat{a}^{\dagger}+\hat{a}\right) \hat{c}^{\dagger} \hat{c} \\
& +\frac{\hbar \omega_{m}}{2}\left(\hat{p}^{2}+\hat{q}^{2}\right)-\hbar G \hat{c}^{\dagger} \hat{c} \hat{q}
\end{aligned}
$$

where $\omega_{m}^{\prime}=4 \omega_{\mathrm{rec}}=4 \frac{\hbar k^{2}}{2 m}, \quad \Delta_{c}=\omega_{c}^{\prime}-\omega_{\mathrm{pu}}$, $\omega_{c}^{\prime}=\omega_{c}+\frac{1}{2} U_{0} N, g=\frac{U_{0}}{2} \sqrt{\frac{N}{2}}$, and $\delta=\omega_{\mathrm{pr}}-\omega_{\mathrm{pu}}$. The first line shows the classical light inputs in the cavity. The first term in the second line gives the energy of cavity mode, $\Delta_{c}$ is the effective Stark-shift detuning, $\frac{1}{2} U_{0} N$ 
denotes the frequency shift of the empty cavity resonance induced by the BEC. The second term in the second line is the energy of Bogoliubov mode of the collective oscillation of the BEC, where $\hat{a}\left(\hat{a}^{\dagger}\right)$ denote the annihilation (creation) operator of the Bogoliubov mode. The third term in the second line describes the coupling energy between the BEC and the cavity, where $g$ is the coupling strength. The last two terms in the third line denote the energy of the mechanical mode and the mirror-cavity interaction.

According to the Heisenberg equation of motion and the communication relation $\left[\hat{a}, \hat{a}^{\dagger}\right]=1,\left[\hat{c}, \hat{c}^{\dagger}\right]=1$, and $[\hat{p}, \hat{q}]=\mathrm{i}$, the evolutions of $\hat{c}, \hat{a}, \hat{p}$, and $\hat{q}$ can be obtained as follows

$$
\begin{aligned}
& \frac{\mathrm{d} \hat{c}}{\mathrm{~d} t}=-\left[\mathrm{i} \Delta_{c}+\kappa-\mathrm{i} G \hat{q}+\mathrm{i} g\left(\hat{a}+\hat{a}^{\dagger}\right)\right] \hat{c} \\
& \quad+E_{\mathrm{pu}}+E_{\mathrm{pr}} \mathrm{e}^{-\mathrm{i} \delta t}+\sqrt{2 \kappa} \hat{c}_{\mathrm{in}}, \\
& \frac{\mathrm{d} \hat{a}}{\mathrm{~d} t}=-\mathrm{i} \omega_{m}^{\prime} \hat{a}-\mathrm{i} g \hat{c}^{\dagger} \hat{c}-\gamma \hat{a}+\sqrt{2 \gamma} \hat{a}_{\mathrm{in}}, \\
& \frac{\mathrm{d} \hat{p}}{\mathrm{~d} t}=\omega_{m} \hat{q}-G \hat{c}^{\dagger} \hat{c}-\gamma_{m} \hat{p}+\hat{\xi}, \\
& \frac{\mathrm{d} \hat{q}}{\mathrm{~d} t}=-\omega_{m} \hat{p} .
\end{aligned}
$$

Here, $\hat{c}_{\text {in }}, \hat{a}_{\text {in }}$, and $\hat{\xi}$ are the corresponding noises operators. Noise operators depend on the reservoir variables. We have introduced the input vacuum noise operators with the zero average values. Then, we deal with the mean response of the system given by equation for the expectation values $\langle c\rangle,\langle p\rangle,\langle q\rangle$, and $\langle X\rangle$ of operators $\hat{c}, \hat{p}, \hat{q}$, and $\hat{X}$ (the latter is defined as $\left.\hat{X}=\left(\hat{a}+\hat{a}^{\dagger}\right) / \sqrt{2}\right)$. These equations are:

$$
\begin{aligned}
& \frac{\mathrm{d}\langle c\rangle}{\mathrm{d} t}=-\left(\mathrm{i} \Delta_{c}+\kappa-\mathrm{i} G\langle q\rangle+\mathrm{i} g\left(\langle a\rangle+\left\langle a^{\dagger}\right\rangle\right)\right)\langle c\rangle \\
& \quad+E_{\mathrm{pu}}+E_{\mathrm{pr}} \mathrm{e}^{-\mathrm{i} \delta t}, \\
& \frac{\mathrm{d}^{2}\langle X\rangle}{\mathrm{d} t^{2}}+\gamma \frac{\mathrm{d}\langle X\rangle}{\mathrm{d} t}+\omega_{m}^{\prime 2}\langle X\rangle=-\omega_{m}^{\prime} g \sqrt{2}\left\langle c^{\dagger}\right\rangle\langle c\rangle, \\
& \frac{\mathrm{d}^{2}\langle q\rangle}{\mathrm{d} t^{2}}+\gamma_{m} \frac{\mathrm{d}\langle q\rangle}{\mathrm{d} t}+\omega_{m}{ }^{2}\langle q\rangle=\omega_{m} G\left\langle c^{\dagger}\right\rangle\langle c\rangle .
\end{aligned}
$$

To solve these equations, we make the following ansatz

$$
\begin{aligned}
& \langle c(t)\rangle=c_{0}+c_{+} \mathrm{e}^{-\mathrm{i} \delta t}+c_{-} \mathrm{e}^{\mathrm{i} \delta t}, \\
& \langle X(t)\rangle=X_{0}+X_{+} \mathrm{e}^{-\mathrm{i} \delta t}+X_{-} \mathrm{e}^{\mathrm{i} \delta t}, \\
& \langle q(t)\rangle=q_{0}+q_{+} \mathrm{e}^{-\mathrm{i} \delta t}+q_{-} \mathrm{e}^{\mathrm{i} \delta t} .
\end{aligned}
$$

Substituting Eq. (6) in Eq. (5), we can obtain:

$$
\begin{aligned}
& c_{0}=\frac{E_{\mathrm{pu}}}{\kappa+\mathrm{i} \Delta_{c}-\mathrm{i} G q_{0}+\mathrm{i} \sqrt{2} g X_{0}}, \\
& c_{+}=\frac{E_{\mathrm{pr}}+\mathrm{i} G q_{+} c_{0}-\mathrm{i} \sqrt{2} g c_{0} X_{+}}{\kappa+\mathrm{i} \Delta_{c}-\mathrm{i} G q_{0}+\mathrm{i} \sqrt{2} g X_{0}-\mathrm{i} \delta}, \\
& c_{-}=\frac{+\mathrm{i} G q_{-} c_{0}-\mathrm{i} \sqrt{2} g c_{0} X_{-}}{\kappa+\mathrm{i} \Delta_{c}-\mathrm{i} G q_{0}+\mathrm{i} \sqrt{2} g X_{0}+\mathrm{i} \delta},
\end{aligned}
$$

$$
\begin{aligned}
& X_{0}=-\frac{\sqrt{2} g\left|c_{0}\right|^{2}}{\omega_{m}^{\prime}}, \\
& X_{+}=-\frac{\omega_{m}^{\prime} \sqrt{2} g\left(c_{0}^{*} c_{+}+c_{-}^{*} c_{0}\right)}{\left(\omega_{m}^{\prime}\right)^{2}-\mathrm{i} \delta \gamma-\delta^{2}}, \\
& X_{-}=-\frac{\omega_{m}^{\prime} \sqrt{2} g\left(c_{0}^{*} c_{-}+c_{+}^{*} c_{0}\right)}{\omega_{m}^{\prime 2}+\mathrm{i} \delta \gamma-\delta^{2}},
\end{aligned}
$$

and

$$
\begin{aligned}
& q_{0}=\frac{G\left|c_{0}\right|^{2}}{\omega_{m}}, \\
& q_{+}=\frac{\omega_{m} G\left(c_{0}^{*} c_{+}+c_{-}^{*} c_{0}\right)}{\omega_{m}^{2}-\mathrm{i} \delta \gamma_{m}-\delta^{2}}, \\
& q_{-}=\frac{\omega_{m} G\left(c_{0}^{*} c_{-}+c_{+}^{*} c_{0}\right)}{\omega_{m}^{2}+\mathrm{i} \delta \gamma_{m}-\delta^{2}},
\end{aligned}
$$

where $c_{0}, X_{0}$, and $q_{0}$ are respectively the intracavity field, BEC, and mechannical displacement for the static solution. From Eqs. (7)-(9), we obtain

$$
\begin{aligned}
& c_{+}=\frac{E_{\mathrm{pr}}\left[(\kappa-\mathrm{i} \delta)-\mathrm{i}\left(\Delta_{c}-C_{1}-C_{2}\right)\right]}{(\kappa-\mathrm{i} \delta)^{2}+\left(\Delta_{c}-C_{1}-C_{2}\right)^{2}-D}, \\
& c_{-}=\frac{\mathrm{i}\left(A_{1} B_{1}^{*} \omega_{m}^{\prime}+A_{2} B_{2}^{*} \omega_{m}\right) E_{\mathrm{pu}}^{2} E_{\mathrm{pr}}}{\left[(\kappa+\mathrm{i} \delta)^{2}+\left(\Delta_{c}-C_{1}^{*}-C_{2}^{*}\right)^{2}-\left(D^{*}\right)^{2}\right] F^{2}},
\end{aligned}
$$
where

$$
\begin{aligned}
& A_{1}=2 g^{2} /\left(\omega_{m}^{\prime}\right)^{2}, \quad A_{2}=G^{2} / \omega_{m}^{2}, \\
& B_{1}=\left(\omega_{m}^{\prime}\right)^{2} /\left[\left(\omega_{m}^{\prime}\right)^{2}-\mathrm{i} \delta \gamma-\delta^{2}\right], \\
& B_{2}=\left(\omega_{m}\right)^{2} /\left[\left(\omega_{m}\right)^{2}-\mathrm{i} \delta \gamma_{m}-\delta^{2}\right], \\
& C_{1}=A_{1} \omega_{m}^{\prime} \omega_{0}\left(B_{1}+1\right), \\
& C_{2}=A_{2} \omega_{m} \omega_{0}\left(B_{2}+1\right), \\
& D=A_{1} B_{1} \omega_{m}^{\prime} \omega_{0}+A_{2} B_{2} \omega_{m} \omega_{0}, \\
& F=\mathrm{i} \Delta_{c}+\kappa-\mathrm{i} A_{1} \omega_{m}^{\prime} \omega_{0}-\mathrm{i} A_{2} \omega_{m} \omega_{0}, \\
& \omega_{0}=\left|c_{0}\right|^{2} .
\end{aligned}
$$

According to Eq. (7)-(9), we can obtain the steady state photon $\omega_{0}$ of the cavity field, phonon number $\left|X_{0}\right|^{2}$ of the collective oscillation of the BEC, and the phonon number $\left|q_{0}\right|^{2}$ of the mechanical resonator:

$$
\begin{aligned}
& E_{\mathrm{pu}}^{2}=\omega_{0}\left[\kappa^{2}+\left(\Delta_{c}-\frac{2 g^{2} \omega_{0}}{\omega_{m}}-\frac{G^{2} \omega_{0}}{\omega_{m}^{\prime}}\right)^{2}\right], \\
& \left|X_{0}\right|^{2}=\frac{2 g^{2} \omega_{0}^{2}}{\omega_{m}^{2}}, \quad\left|q_{0}\right|^{2}=\frac{G^{2} \omega_{0}^{2}}{\left(\omega_{m}^{\prime}\right)^{2}},
\end{aligned}
$$

Therefore, the steady-state photon number $\omega_{0}$ of the cavity field, phonon number $\left|X_{0}\right|^{2}$ of the collective oscillation of the BEC, and the phonon number $\left|q_{0}\right|^{2}$ of the mechanical resonator depend on each other. This form of coupled equations are characteristic of optical multistability.

We use then the input-output relation which is valid for one-sided open cavity: 


$$
c_{\text {out }}(t)=c_{\text {in }}(t)-\sqrt{2 \kappa} c(t),
$$

where $c_{\text {in }}$ and $c_{\text {out }}$ are the input and output operators. For our hybrid optomechanical systems, we can obtain the output field

$$
\begin{aligned}
& c_{\text {out }}(t)=\left[\left(E_{\mathrm{pu}} / \sqrt{2} \kappa\right)-\sqrt{2} \kappa c_{0}\right] \mathrm{e}^{-\mathrm{i} \omega_{\mathrm{pu}} t} \\
& +\left(E_{p r} / \sqrt{2} \kappa-\sqrt{2} \kappa c_{+}\right) \mathrm{e}^{-\mathrm{i}\left(\omega_{\mathrm{pu}}+\delta\right) t} \\
& -\sqrt{2} \kappa c_{-} \mathrm{e}^{-\mathrm{i}\left(\omega_{\mathrm{pu}}-\delta\right) t}
\end{aligned}
$$

where $c_{\text {out }}(t)$ is the output field operator in the original frame. The output fields contain two input components (the driven field $\omega_{\mathrm{pu}}$ and the probe field $\omega_{\mathrm{pr}}$ ) and one generated FWM component at the frequency $2 \omega_{\mathrm{pu}}-\omega_{\mathrm{pr}}$ in Eq. (14). The transmission of the probe field is defined as

$$
\begin{aligned}
& t_{p}=\frac{E_{\mathrm{pr}} / \sqrt{2} \kappa-\sqrt{2} \kappa c_{+}}{E_{\mathrm{pr}} / \sqrt{2} \kappa}= \\
& 1-\frac{2 \kappa\left[(\kappa-\mathrm{i} \delta)-\mathrm{i}\left(\Delta_{c}-C_{1}-C_{2}\right)\right]}{(\kappa-\mathrm{i} \delta)^{2}+\left(\Delta_{c}-C_{1}-C_{2}\right)^{2}-D} .
\end{aligned}
$$

The relative transmitted intensity of the four wave mixing defined as the ratio of the output and input field amplitudes at the frequency is then given by

$$
\begin{aligned}
& \left|T_{\text {out }-}\right|^{2}=\left|\frac{c_{-}}{E_{\mathrm{pr}} / \sqrt{2 \kappa}}\right|^{2}= \\
& \left|\frac{2 \kappa\left(A_{1} B_{1}^{*} \omega_{m}^{\prime}+A_{2} B_{2}^{*} \omega_{m} E_{\mathrm{pu}}^{2}\right)}{\left[(\kappa+\mathrm{i} \delta)^{2}+\left(\Delta_{c}-C_{1}^{*}-C_{2}^{*}\right)^{2}-\left(D^{*}\right)^{2}\right] F^{2}}\right|^{2} .
\end{aligned}
$$

\section{Results and discussion}

In this section, we first discuss the optical bistable behavior of the steady-state photon number $\omega_{0}$ of the cavity field, phonon number $\left|X_{0}\right|^{2}$ of the collective oscillation of the BEC, and the phonon number $\left|q_{0}\right|^{2}$ of the mechanical resonator according to Eq. (12). Furthermore, we investigate the four wave mixing based on the numerical solutions of Eq. (15) and (16). Finally, we also demonstrate the four wave mixing intensity affected by the pump strength, the frequency difference between the BEC and the moving end mirror, cavity decay rate, and effective coupling strength of the optical field with the moving mirror.

In order to better understand the optical bistable behavior and tunable four-wave mixing based on hybrid BEC-optomechanical systems, we choose the realistic parameters of the hybrid optomechanical systems as follows [27, 28]. We consider $N=2.3 \times 10^{4}$ atoms of ${ }^{87} \mathrm{Rb}$, trapped inside Fabry-Pérot cavity with length $L=1.25 \times 10^{-4} \mathrm{~m}$, driven by the single mode external field with wavelength $\lambda_{p}=780 \mathrm{~nm}$. The intra-cavity optical mode has decay rate $\kappa=2 \pi \times 1.3 \mathrm{kHz}$. Further, intra-cavity field produces recoil of $\omega_{\text {rec }}=2 \pi \times 3.8 \mathrm{kHz}$ in atomic mode trapped inside cavity with damping rate $\gamma=0.001 \kappa$. The moving end mirror of cavity is considered as a perfect reflector oscillating with frequency $\omega_{m}=1.1 \omega_{m}^{\prime}$ with damping $\gamma_{m}=0.001 \kappa$. In the following conditions the optical cavity is driven by a red detuned laser field, and in the resolved sideband condition, $\omega_{m} \gg \kappa$, which ensures the normal mode splitting to be distinguished.

We first discuss the optical bistable behavior of the hybrid optomechanical systems. In Fig. 2, we have plotted the mean number of photons $\omega_{0}$ as the cavity pump detuning $\Delta_{c}$ for four different values of the interaction of mirror-cavity: $\mathrm{G}=0$ (Fig. 2a), $\mathrm{G}=\mathrm{g}$ (Fig. 2b), $\mathrm{G}=2 \mathrm{~g}$ (Fig. 2c), and $\mathrm{G}=3 \mathrm{~g}$ (Fig. 2d), respectively. In order to see the effect of the pump strength on the optical bistability of the system, we have plotted the mean photon number of the system in three different pump strengths: $\quad E_{p u}=0.1 \mathrm{kHz}$ (the black dot line), $E_{\mathrm{pu}}=0.3 \mathrm{kHz}$ (the blue dot line), and $E_{\mathrm{pu}}=0.4 \mathrm{kHz}$ (the red dot line). In Fig. 2a, the max power of the laser pump is $E_{\mathrm{pu}}=0.4 \mathrm{kHz}$, which is far below the bistability threshold of the system. As is seen, all curves have the same form in the absence of the interaction of mirror cavity, and there is no bistable phenomenon. Increasing the interaction of mirror-cavity cause the system to approach the bistability region. In Figure 2b, where the power of the laser pump is the same as Fig. 2a, which is below the bistability threshold of the system, so the curves are the same as Fig. 2a. In contrast, in Fig. 2c and d, their bistability regions become clear with increase in the interaction of mirrorcavity. In short, on one hand the interaction of mirrorcavity of the hybrid optomechanical cavity causes the system to become bistable at lower pump powers. On the other hand, the optical bistability becomes larger and the bistable regime becomes wider with increase in the pump powers and with increase in the interaction of mirror-cavity.

Furthermore, we can see from the Eq. (12), that the phonon number $\left|X_{0}\right|^{2}$ of the collective oscillation of the BEC and the phonon number $\left|q_{0}\right|^{2}$ of the mechanical resonator are related to the steady-state photon number $\omega_{0}$ of the cavity field. Figure 3 shows the steady state phonon number $\left|X_{0}\right|^{2}$ of the collective oscillation of the BEC and the steady-state phonon number $\left|q_{0}\right|^{2}$ of the mechanical resonator as a function of cavity-pump detuning $\Delta_{c}$ for three different values of pump strengths $E_{\mathrm{pu}}=0.1 \mathrm{kHz}$ (the black dot line), $E_{\mathrm{pu}}=0.3 \mathrm{kHz}$ (the blue dot line), and $E_{\mathrm{pu}}=0.4 \mathrm{kHz}$ (the red dot line) with the interaction of mirror-cavity $G=2 g$. For the fixed interaction of mirror-cavity, the optical bistable behavior of the steady-state phonon number $\left|X_{0}\right|^{2}$ and the phonon number $\left|q_{0}\right|^{2}$ can also occur with function of cavity-pump detuning $\Delta_{c}$, which is similar to the curve for $G=2 g$ in Fig. 2(c). Furthermore, the two curves for the phonon number $\left|X_{0}\right|^{2}$ and $\left|q_{0}\right|^{2}$ in Fig. 3a and $\mathrm{b}$ coincide. The reason can be explained by Eq. (12). Phonon number $\left|X_{0}\right|^{2}$ of the collective oscillation of the BEC and the phonon number $\left|q_{0}\right|^{2}$ of the mechanical resonator depends on the steady-state photon number $\omega_{0}$ of the cavity field. 

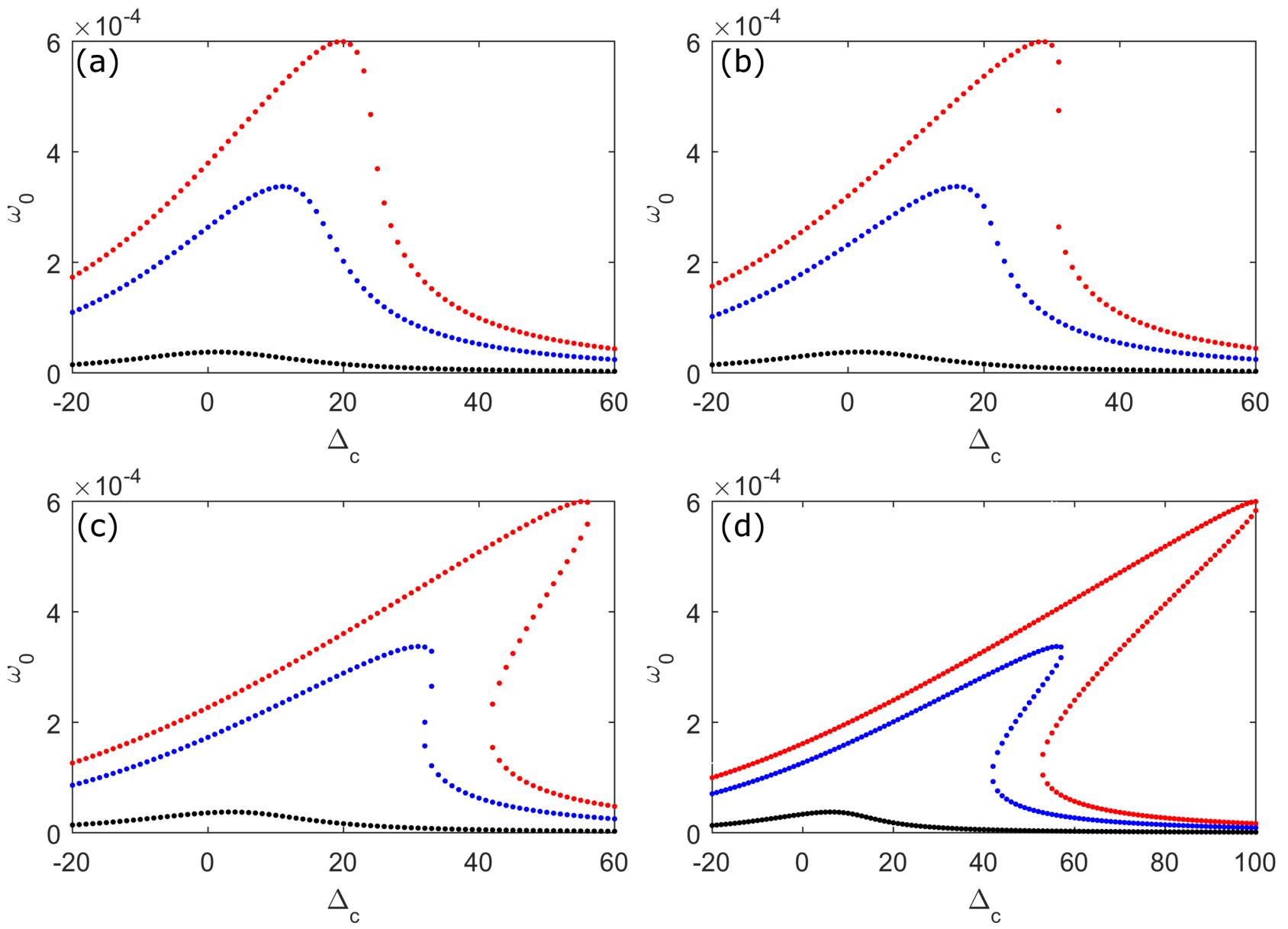

Fig. 2. The mean photon number $\omega_{0}$ of the intracavity as a function of cavity-pump detuning $\Delta_{c}$ for three different values of pump strengths $E_{\mathrm{pu}}=0.1 \mathrm{kHz}$ (the black dot line), $E_{\mathrm{pu}}=0.3 \mathrm{kHz}$ (the blue dot line) and $E_{\mathrm{pu}}=0.4 \mathrm{kHz}$ (the red dot line), calculated for four different values of the interaction of mirror-cavity: (a) $G=0$, (b) $G=g$, (c) $G=2 g$ and (d) $\mathrm{G}=3 \mathrm{~g}$, respectively. The other parameters are $g_{0}=2 \pi \times 10.9 \mathrm{MHz}, N=2.3 \times 10^{4}, \omega_{m}=2 \pi \times 15.2 \mathrm{kHz}$, $\omega_{m}^{\prime}=1.1 \omega_{m}, \Delta_{a}=2 \pi \times 32 \mathrm{GHz}, \kappa=2 \pi \times 1.3 \mathrm{kHz}$ and $\gamma=\gamma_{m}=0.001 \kappa$.

In Fig. 4, we plot the probe transmission $\left|t_{p}\right|^{2}$ and the FWM intensity as a function of the normalized frequency $\delta / \bar{\omega}$ with $G=0$ and $G=g$. In the absence of the effective coupling strength of the optical field with the mirror, i.e., $G=0$, it can be seen from Fig. $4 \mathrm{a}$ that OMIT appears in the center of the normalized frequency $\delta / \bar{\omega}$, which can be well explained in terms of the radiation pressure force oscillating at the beat frequency $\Delta_{c}=\bar{\omega}$ between the probe and control field. Furthermore, we find that FWM peak can be greatly enhanced under the condition of OMIT, as shown in Fig. $4 \mathrm{~b}$. There is a peak at the $\delta / \bar{\omega}=1$ in the FWM spectrum. When the effective coupling strength of the optical field with the mirror turns on $(G=g)$, the transparency window at the $\delta / \bar{\omega}=1$ is split into two transparency windows, which yields the double-OMIT. Correspondingly, two peaks appear in Fig. 4(b) at the positions of the two transparency windows, respectively. The system is pumped by the lower pump laser field. The collective motion of the BEC can be performed as an analogy to a mechanical resonator, and the moved mirror as a mechanical resonator. The physical effect can be explained by OMIT, which originates from the radiation pressure coupling an optical mode to a mechanical mode. The OMIT depends on quantum interference. The coupling between the optical field with the condensate mode and the mirror breaks down the symmetry of the OMIT interference. Then, the single OMIT window is split into two transparency windows.

To determine the strength of pump laser affected on the FWM intensity, we plot the FWM intensity as function of the normalized frequency $\delta / \bar{\omega}$ for different values of pump strength $E_{\mathrm{pu}}$. They are indicated with color lines in Fig. 5 , where $E_{\mathrm{pu}}=0.05 \mathrm{kHz}$ (black), $E_{\mathrm{pu}}=0.1 \mathrm{kHz}$ (blue), and $E_{\mathrm{pu}}=0.3 \mathrm{kHz}$ (red). It can be seen that the peak FWM intensity first increases quickly and reaches a maximum value with increase in the strength of the pump laser. Furthermore, we also find that the distance of the window becomes larger as the pump strength increases. The physical reason for this result is related to the nonlinearity of the hybrid optomechanical system. When the pump strength 

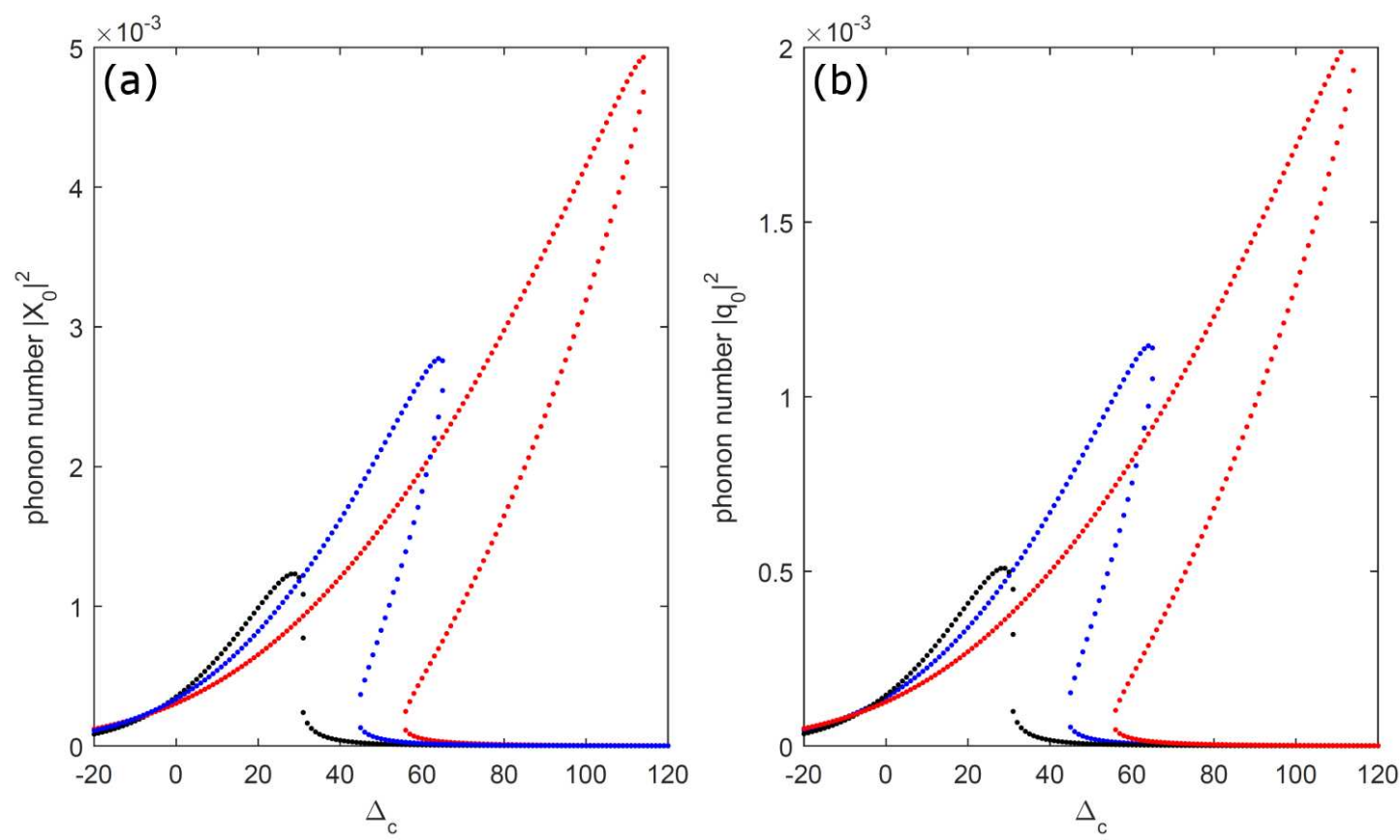

Fig. 3. (a) the steady state phonon number $\left|X_{0}\right|^{2}$ of the collective oscillation of the BEC and (b) the steady-state phonon number $\left|q_{0}\right|^{2}$ of the mechanical resonator as a function of cavity-pump detuning $\Delta_{c}$ with the interaction of mirror-cavity $G=2 g$ for three different values of pump strengths $E_{\mathrm{pu}}=0.1 \mathrm{kHz}$ (the black dot line), $E_{\mathrm{pu}}=0.3 \mathrm{kHz}$ (the blue dot line), and $E_{\mathrm{pu}}=0.4 \mathrm{kHz}$ (the red dot line). The other parameters are the same as those of Fig. 2.
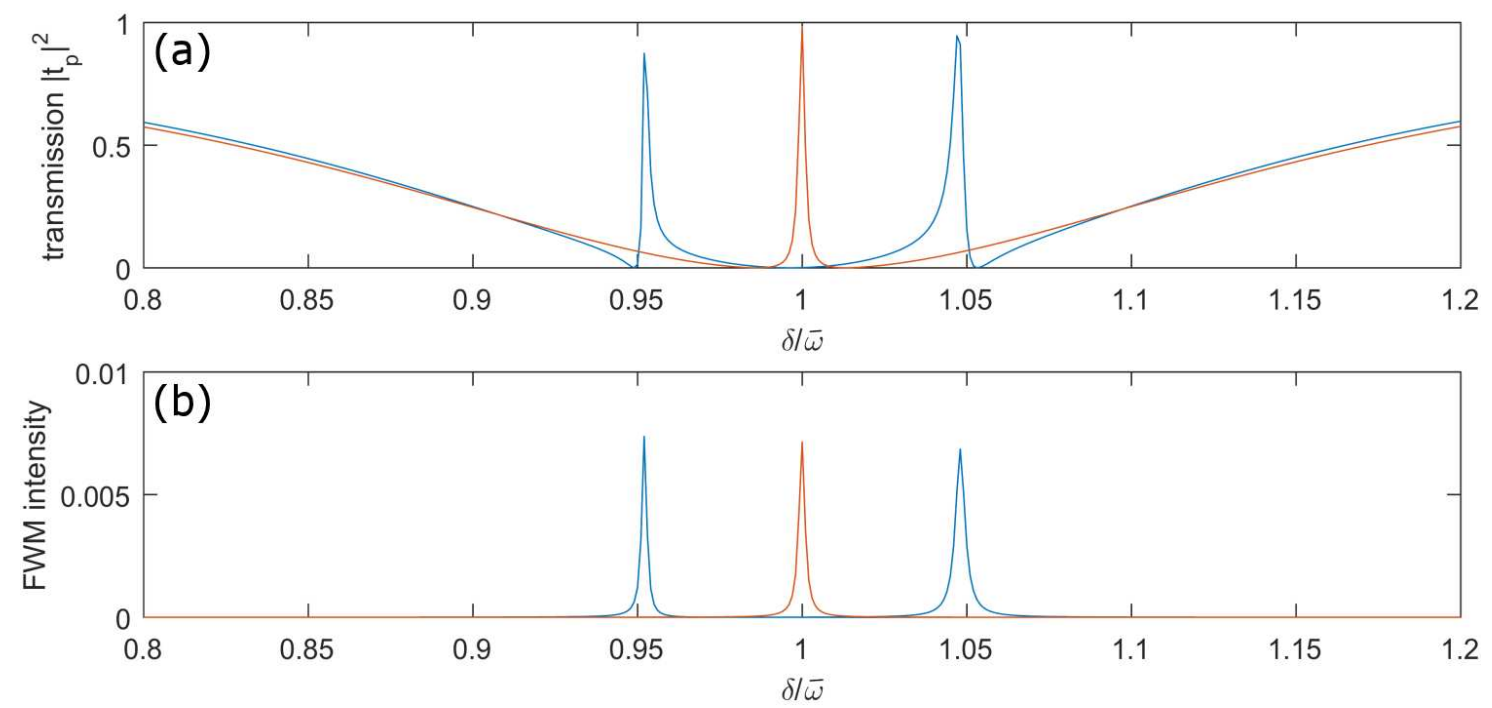

Fig. 4. (a) The probe transmission spectum and (b) FWM intensity $\left|T_{\text {out }-}\right|^{2}$ as a function of $\delta / \bar{\omega}$, with $\Delta_{c}=\bar{\omega}$ for $G=1 g$ (the blue line) and $G=0$ (the brown line). The other parameters are the same as those of Fig. 2.

is relatively small, more intracavity photons are stimulated when pump strength increases and thus the effective coupling strength between the cavity and the mechanical resonator gets stronger. The probe field and the control field can therefore mix efficiently via the effective coupling of the optical field with the condensate mode and the effective coupling of the optical field with the moved mirror.
Furthermore, we investigate the effect of frequency difference between the BEC and the moving end mirror on FWM intensity $\left|T_{\text {out }-}\right|^{2}$. The resulting spectra are shown in Fig. 6. One can find there is only one peak when the frequencies of BEC and the movable mirror are same, i.e. $\omega_{m}=\omega_{m}^{\prime}$. In the case of $\omega_{m} \neq \omega_{m}^{\prime}$, FWM intensity $\left|T_{\text {out }-}\right|^{2}$ exhibits other two peaks at $\omega=\bar{\omega}+\omega_{0}$ and $\omega=\bar{\omega}-\omega_{0}$. The small deviation $\omega_{0}$ from the central 


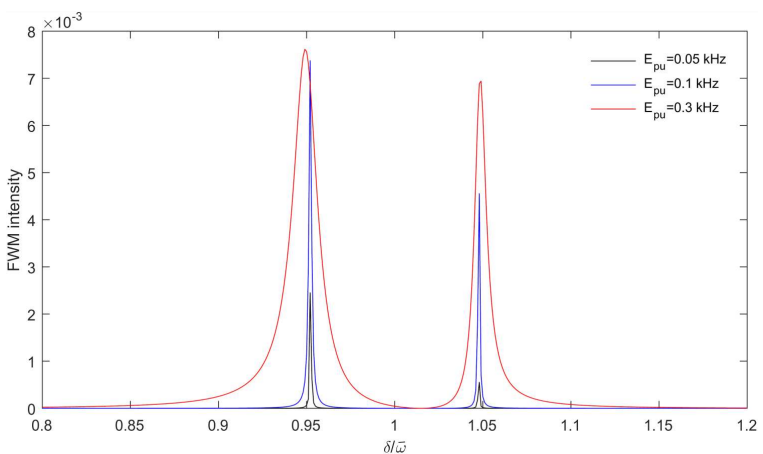

Fig. 5. FWM intensity $\left|T_{\text {out }-}\right|^{2}$ as a function of $\delta / \bar{\omega}_{m}$, with $\Delta_{c}=\bar{\omega}$ for $E_{\mathrm{pu}}=0.05 \mathrm{kHz}$ (the black line), $E_{\mathrm{pu}}=$ $0.1 \mathrm{kHz}$ (the blue line) and $E_{\mathrm{pu}}=0.3 \mathrm{kHz}$ (the red line). The other parameters are the same as those of Fig. 2.

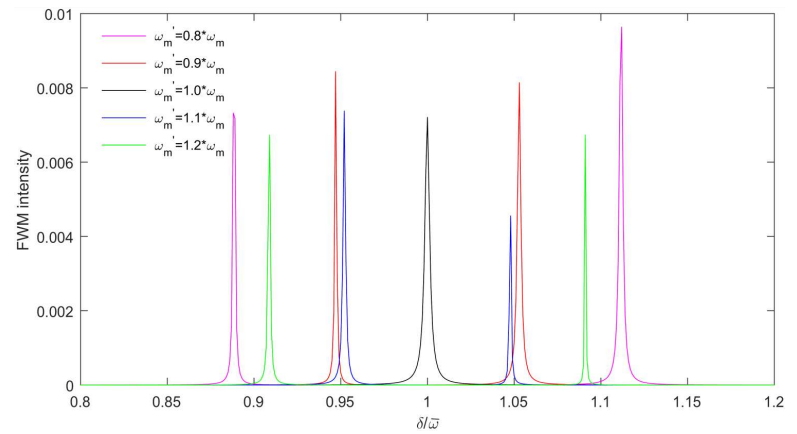

Fig. 6. FWM intensity $\left|T_{\text {out }-}\right|^{2}$ as a function of $\delta / \bar{\omega}$, with $\Delta_{c}=\bar{\omega}$ for $\omega_{m}=0.8 \omega_{m}^{\prime}, \omega_{m}=0.9 \omega_{m}^{\prime}, \omega_{m}=\omega_{m}^{\prime}$, $\omega_{m}=1.1 \omega_{m}^{\prime}$, and $\omega_{m}=1.2 \omega_{m}^{\prime}$. The other parameters are the same as those of Fig. 2.

frequency $\bar{\omega}$ depends on the frequency difference between the BEC and the moving end mirror. If $\omega_{m}^{\prime}<\omega_{m}$, two normal peaks are further and further apart with the increase of the frequency difference between the BEC and the moving mirror. If $\omega_{m}^{\prime}>\omega_{m}$, two normal peaks are further and further apart with the decrease of the frequency difference between the BEC and the moving mirror.

Now, we are going to study the influence of the cavity decay rate $\kappa$ on the FWM intensity $\left|T_{\text {out }-}\right|^{2}$. We analyze it in Fig. 7 for different values of cavity decay, namely $\kappa=1.3 \pi \mathrm{kHz}$ (the black line), $\kappa=2 \pi \times 1.3 \mathrm{kHz}$ (the blue line), and $\kappa=4 \pi \times 1.3 \mathrm{kHz}$ (the red line). It can be seen that the peak FWM intensity first increases with increase in the cavity decay rate $\kappa$, while the positions of the peaks of these curves remain unchanged.

Finally, in Fig. 8, we plot the FWM intensity $\left|T_{\text {out }-}\right|^{2}$ as function of the normalized frequency $\delta / \bar{\omega}$ for different effective coupling strengths of the optical field with the moving mirror for $G=0$ (the black line), $G=g$ (the blue line), $G=2 g$ (the green line), and $G=3 g$ (the red line). In the absence of effective coupling strength of the optical field with the moving mirror, the strength of the four wave mixing has only one peak. While in the presence of effective coupling strength of the optical field with the moving mirror, there are two peaks in the FWM intensity $\left|T_{\text {out- }}\right|^{2}$. Furthermore, the line widths of the peaks increase with increase in the effective coupling strength of the optical field with the moving mirror.

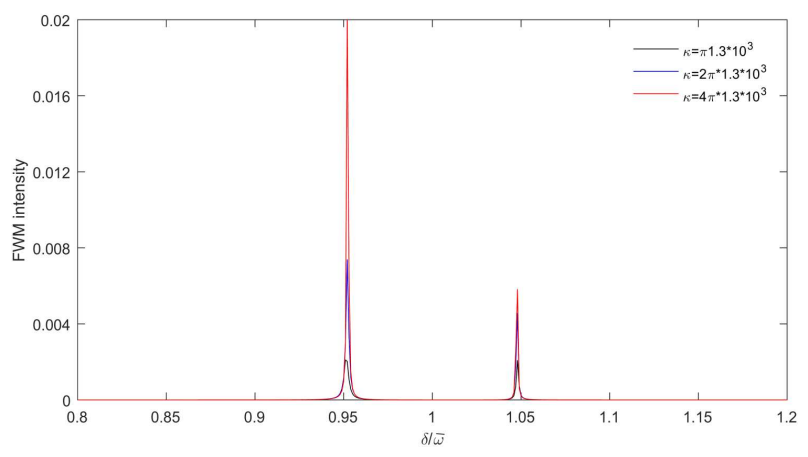

Fig. 7. FWM intensity $\left|T_{\text {out }-}\right|^{2}$ as a function of $\delta / \bar{\omega}$ for $\kappa=1.3 \pi \mathrm{kHz}$ (the black line), $\kappa=2 \pi \times 1.3 \mathrm{kHz}$ (the blue line) and $\kappa=4 \pi \times 1.3 \mathrm{kHz}$ (the red line). The other parameters are the same as those of Fig. 2.

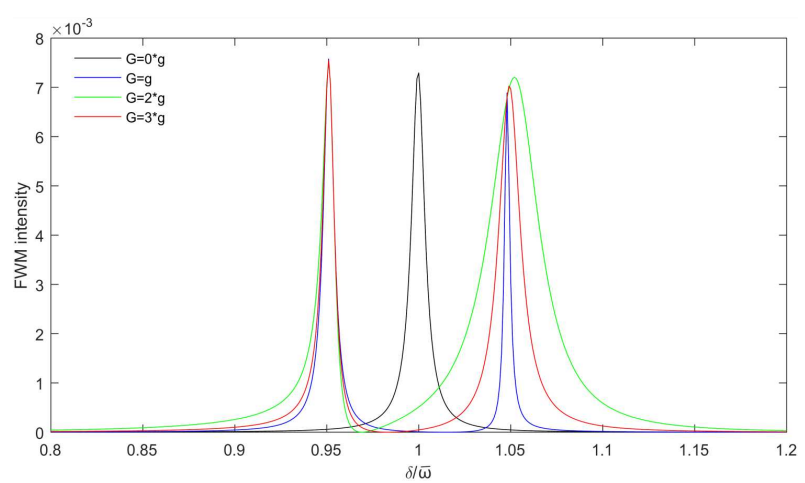

Fig. 8. FWM intensity $\left|T_{\text {out }-}\right|^{2}$ as a function of $\delta / \bar{\omega}$ for $G=0$ (the bleak line), $G=g$ (the blue line), $G=2 g$ (the green line) and $G=3 g$ (the red line). The other parameters are the same as those of Fig. 2.

\section{Added noises of the FWM}

To deal with Eq. (4), we use the Fourier transform: $f(t)=\frac{1}{2 \pi} \int_{-\infty}^{\infty} f(\omega) \mathrm{e}^{-\mathrm{i} \omega t} \mathrm{~d} \omega$, and $f^{\dagger}(t)=$ $\frac{1}{2 \pi} \int_{-\infty}^{\infty} f^{\dagger}(-\omega) \mathrm{e}^{-\mathrm{i} \omega t} \mathrm{~d} \omega$ that is valid for any operator $f(t) \in\{\delta c, \delta X, \delta p, \delta q\}$. Here, $\delta X$ is treated as $\hat{X}=$ $\left(\hat{a}+\hat{a}^{\dagger}\right) / \sqrt{2}$.

Now, Eq. (4) can be rewritten as

$$
\begin{aligned}
& -\mathrm{i} \omega \delta c(\omega)=-(\mathrm{i} \Delta+\kappa) \delta c(\omega)-\mathrm{i} g c_{0} \delta X(\omega) \\
& +\mathrm{i} G c_{0} \delta q(\omega)+\sqrt{2 \kappa} c_{\mathrm{in}}(\omega), \\
& -\mathrm{i} \omega \delta X(\omega)=\omega_{m}^{\prime} \delta Y(\omega)-\gamma \delta X(\omega),
\end{aligned}
$$




$$
\begin{gathered}
-\mathrm{i} \omega \delta Y(\omega)=-\omega_{m}^{\prime} \delta X(\omega)-\gamma \delta Y(\omega) \\
-g\left(c_{0} \delta c^{\dagger}(-\omega)+c_{0}{ }^{*} \delta c(\omega)\right)+f_{m}(\omega), \\
-\mathrm{i} \omega \delta p(\omega)=-\omega_{m} \delta q(\omega)-\gamma_{m} \delta Y(\omega) \\
+G\left(c_{0} \delta c^{\dagger}(-\omega)+c_{0}^{*} \delta c(\omega)\right)+\xi(\omega), \\
-\mathrm{i} \omega \delta q(\omega)=\omega_{m} \delta p(\omega),
\end{gathered}
$$

The operators $c_{\text {in }}(\omega), f_{m}(\omega)$, and $\xi(\omega)$ denote the corresponding noises zero average value. These noise operators satisfy the following correlation function [18]:

$$
\begin{aligned}
& \left\langle c_{\mathrm{in}}(\omega) c_{\mathrm{in}}(-\omega)\right\rangle=1, \\
& \left\langle f_{m}(\omega) f_{m}(-\omega)\right\rangle=\gamma_{s m}, \\
& \langle\xi(\omega) \xi(-\omega)\rangle=\frac{\gamma_{m}}{\omega}\left[1+\operatorname{coth}\left(\frac{\hbar \omega}{2 k_{\mathrm{B}} T}\right)\right] .
\end{aligned}
$$

Above equations, further, give the following solution:

$$
\begin{aligned}
& \delta c(\omega)=E(\omega) c_{\text {in }}(\omega)+F(\omega) c_{\text {in }}^{\dagger}(-\omega) \\
& +V_{1} f_{m}(\omega)+V_{2} \xi(\omega),
\end{aligned}
$$

in which

$$
\begin{aligned}
& E(\omega)=\sqrt{2 \kappa} \frac{\mathrm{i}\left(\lambda_{1}+\lambda_{2}\right)+(\kappa-\mathrm{i} \Delta-\mathrm{i} \omega)}{d(\omega)}, \\
& F(\omega)=\sqrt{2 \kappa} \frac{\mathrm{i}\left(\lambda_{1}+\lambda_{2}\right)}{d(\omega)}, \\
& V_{1}=\frac{-\mathrm{i} g c_{0}(\kappa-\mathrm{i} \Delta-\mathrm{i} \omega)\left(\gamma-\mathrm{i} \omega+\omega_{m}^{\prime}\right)}{d(\omega)\left(\omega_{m}^{\prime}{ }^{2}+(\gamma-\mathrm{i} \omega)^{2}\right)}, \\
& V_{2}=\frac{\mathrm{i} G \omega_{m}(\kappa-\mathrm{i} \Delta-\mathrm{i} \omega)}{d(\omega)\left(\omega_{m}^{2}-\omega^{2}-\mathrm{i} \gamma_{m} \omega\right)},
\end{aligned}
$$

with

$$
\begin{aligned}
& \lambda_{1}=\frac{\eta^{2}\left(\omega_{m}^{\prime}\right)}{\left(\omega_{m}^{\prime}\right)^{2}+(\gamma-\mathrm{i} \omega)^{2}}, \quad \lambda_{2}=\frac{\zeta^{2} \omega_{m}}{\omega_{m}^{2}-\omega^{2}-\mathrm{i} \gamma_{m} \omega}, \\
& d(\omega)=(\kappa-\mathrm{i} \omega)^{2}+\Delta^{2}-2 \Delta\left(\lambda_{1}+\lambda_{2}\right),
\end{aligned}
$$

where $\eta=g\left|c_{0}\right|, \zeta=G\left|c_{0}\right|$ are the effective coupling of the optical field with the condensate mode and the moving-end mirror. Then, using the input-output relation $c_{\text {out }}(\omega)=\sqrt{2 \kappa} c(\omega)-c_{\text {in }}(\omega)$, defining the spectrum of the field via $\left\langle\delta c^{\dagger}(-\Omega) \delta c(\omega)\right\rangle=2 \pi S_{c}(\omega) \delta(\omega+\Omega)$, $\left\langle\delta c(-\Omega) \delta c^{\dagger}(\omega)\right\rangle=2 \pi\left(S_{c}(\omega)+1\right) \delta(\omega+\Omega)$. We can obtain the spectrum of the output fields

$$
\begin{aligned}
& S_{\text {cout }}(\omega)=R(\omega) S_{\text {cin }}+S^{v}(\omega)+S_{1}(\omega)+S_{2}(\omega), \\
& S_{\text {dout }}(\omega)=T(\omega) S_{\text {cin }}+S^{v}(\omega)+S_{1}(\omega)+S_{2}(\omega),
\end{aligned}
$$
where

$$
\begin{aligned}
& R(\omega)=|\sqrt{2 \kappa} E(\omega)-1|^{2}, \quad T(\omega)=|\sqrt{2 \kappa} E(\omega)|^{2}, \\
& S^{v}(\omega)=2|\sqrt{2 \kappa} F(\omega)|^{2}, \quad S_{1}^{T}(\omega)=\gamma\left|V_{1}(\omega)\right|^{2}, \\
& S_{2}^{T}(\omega)=\frac{\gamma_{m}}{\omega}\left[1+\operatorname{coth}\left(\frac{\hbar \omega}{2 k_{\mathrm{B}} T}\right)\right]\left|V_{2}(\omega)\right|^{2} .
\end{aligned}
$$

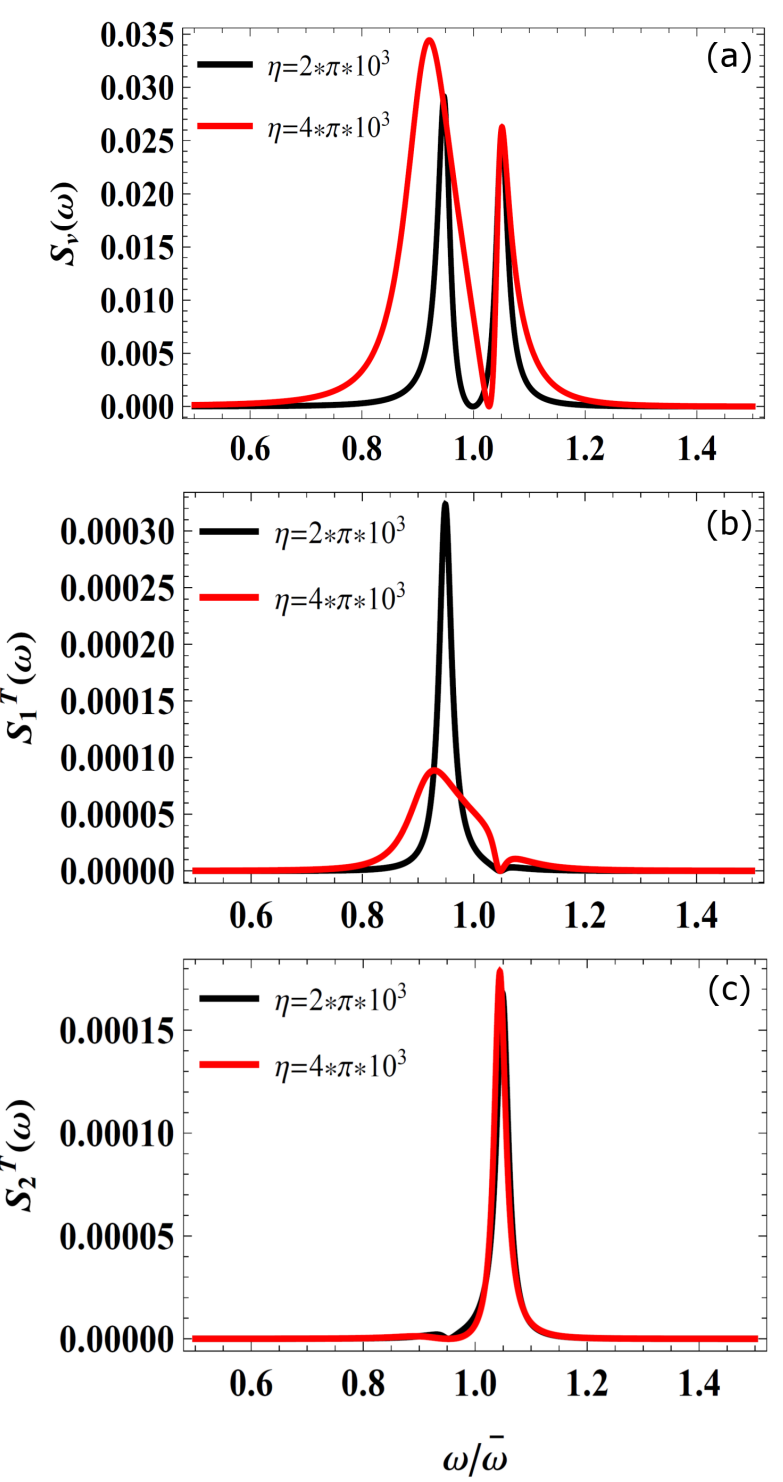

Fig. 9. (a) The vacuum noise spectrum $S_{v}(\omega)$. (b) the thermal noise spectrum $S_{1}^{T}(\omega)$ and (c) $S_{2}^{T}(\omega)$ as function of the normalized frequency $\omega / \bar{\omega}$ for the effective coupling of the optical field with the condensate mode $\eta=2 \pi \mathrm{kHz}$, (black curve), and $\eta=4 \pi \mathrm{kHz}$ (red curve). The other parameters are: $\omega_{m}=2 \pi \times 15.2 \mathrm{kHz}$, $\omega_{m}^{\prime}=1.1 \omega_{m}, \Delta=\left(\omega_{m}+\omega_{m}^{\prime}\right) / 2, \zeta=2 \pi \mathrm{kHz}$ and $\gamma_{s m}=\gamma_{m}=0.001 \kappa$.

In Eq. (22), $R(\omega)$ and $T(\omega)$ are the contributions arising from the presence of the input field. $S^{v}(\omega)$ is contribution of the nature of the vacuum field. $S_{1}^{T}(\omega)$ and $S_{2}^{T}(\omega)$ are the contributions from the fluctuations of the effective mechanical oscillator mode.

Finally, we discuss the effects of the vacuum noise spectrum $S_{v}(\omega)$ and the thermal noise spectrum $S_{1}^{T}(\omega)$ and $S_{2}^{T}(\omega)$ as functions of the normalized frequency $\omega / \bar{\omega}$ for the effective coupling of the optical field with the condensate mode $\eta=2 \pi \mathrm{kHz}$ (black curve), and $\eta=4 \pi \mathrm{kHz}$ (red curve) are shown in Fig. 9. We can see the behavior 
of the vacuum noise $S_{v}(\omega)$ for two different values of the effective coupling of the optical field with the condensate mode. The maximum contribution of the noises is about $0.35 \%$, which is insignificant. For the thermal noise $S_{1}^{T}(\omega)$ and $S_{2}^{T}(\omega)$ for two different values of the effective coupling of the optical field with the condensate mode are shown in Figs. 9b and c. One can find that for larger pumping field powers, $S_{1}^{T}(\omega)$ split into two separate peaks. The maximum contribution of the thermal noise is about $0.3 \%$, which is insignificant too. However, if we work with mirror temperatures like $20 \mathrm{mK}$, then the thermal noise term is insignificant as shown in Fig. 9 [18, 33].

\section{Conclusion}

We have investigated the optical bistability and fourwave mixing in a hybrid optomechanical systems consisting of a Bose-Einstein condensate trapped inside an optical cavity with a moving end-mirror. Numerical results show that the optical bistability can be adjusted by the control pump strengths and effective coupling strength of the optical field with the moving mirror. Moreover, the output fields of the cavity are significantly modified by effective coupling strength of the optical field with the condensate mode and effective coupling strength of the optical field with the moving mirror. Two transparency windows exist in the transmission spectrum of the probe field, which can further enhance the intensity of the four-wave mixing field with reduced linear absorption. The splitting distance between the two peaks in the FWM spectrum can be controlled by the frequency difference between the BEC and the moving end mirror. Furthermore, the relationship between the peak value of the FWM intensity can be controlled effectively by the pump strength, the frequency difference between the BEC and the moving end mirror, cavity decay rate, and effective coupling strength of the optical field with the moving mirror. Finally, we also demonstrate the vacuum and thermal noise can be insignificant for the FWM spectrum.

\section{Acknowledgments}

Project supported by the National Natural Science Foundation of China (Grant No. 11564034, No. 11764036, No. 21663026), the Fundamental Research Funds for the Central Universities of College of Electrical Engineering, Northwest Minzu University (No. 31920180021), the Scientific research funds of College of Electrical Engineering, Northwest Minzu University (No. xbmuyjrc201115).

\section{References}

[1] T.J. Kippenberg, K.J. Vahala, Science 321, 1172 (2008).

[2] M. Aspelmeyer, T.J. Kippenberg, F. Marquardt, Rev. Mod. Phys. 86, 1391 (2014).
[3] Y. Turek, Y. Li, C.P. Sun, Phys. Rev. A 88, 053827 (2013).

[4] S. Huang, G.S. Agarwal, Phys. Rev. A 83, 023823 (2011).

[5] Q. Zheng, Y. Yao, Y. Li, Phys. Rev. A 93, 013848 (2016).

[6] F. Brennecke, S. Ritter, T. Donner, T. Esslinger, Science 322, 235 (2008).

[7] A. Dalafi, M.H. Naderi, A. Motazedifard, Phys. Rev. A 97, 043619 (2018).

[8] R. Kanamoto, P. Meystre, Phys. Rev. Lett. 104, 063601 (2010).

[9] T.P. Purdy, D.W.C. Brooks, T. Botter, N.B.Z.Y. Ma, D.M. Stamper-Kurn, Phys. Rev. Lett. 105, 133602 (2010).

[10] E.A. Sete, H. Eleuch, Phys. Rev. A 85, 043824 (2012).

[11] W. Xiong, D.Y. Jin, Y.Y. Qiu, C. Lam, J.Q. You, Phys. Rev. A 93, 023844 (2016).

[12] A. Dalafi, M. H. Naderi, Phys. Rev. A 95, 043601 (2017).

[13] S. Weis, R. Rivière , S. Deléglise, E. Gavartin, O. Arcizet, A. Schliesser, T.J.O. Kippenberg, Science 330, 1520 (2010).

[14] A.H. Safavi-Naeini, T.P.M. Alegre, M.E.J. Chan, M. Winger, Q. Lin, J.T. Hill, D.E. Chang, O. Painter, Nature 472, 69 (2011).

[15] F. Massel, T.T. Heikkilä, J.M. Pirkkalainen, H.S.S. Cho, P.J. Hakonen, M.A. Sillanpää, Nature (London) 480, 351 (2011).

[16] M. Karuza, C. Biancofiore, M. Bawaj, C. Molinelli, M. Galassi, R. Natali, P. Tombesi, G.D. Giuseppe, D. Vitali, Phys. Rev. A 88, 013804 (2013).

[17] S.M. Huang, G.S. Agarwal, Phys. Rev. A 81, 033830 (2010).

[18] G.S. Agarwal, S.M. Huang, Phys. Rev. A 85 021801(R) (2012).

[19] J.D. Teufel, D. Li, M.S. Allman, K. Cicak1, A.J. Sirois, J.D. Whittaker, R.W. Simmonds, Nature 471, 204 (2011).

[20] D. Tarhan, S.M. Huang, O.E. Müstecaplioğlu, Phys. Rev. A 87, 013824 (2013).

[21] J.Q. Zhang, Y. Li, M. Feng, Y. Xu, Phys. Rev. A 86, 053806 (2012).

[22] K.A. Yasir, W.M. Liu, Sci. Rep. 5, 10612 (2015).

[23] K.A. Yasir, W.M. Liu, Sci. Rep. 6, 22651 (2016).

[24] Y. Li, M. Xiao, Opt. Lett. 21, 1064 (1996).

[25] H. Schmidt, K.L. Campman, A.C. Gossard, A. Imamoğlu, Appl. Phys. Lett. 70, 3455 (1997).

[26] B. Chen, C. Jiang, K.D. Zhu, J. Opt. Soc. Am. B 28, 2007 (2011).

[27] F. Brennecke, T. Donner, S. Ritter, T. Bourdel, M. Köhl, T. Esslinger, Nature 450, 268 (2007).

[28] Y. Colombe, T. Steinmetz, G. Dubois, F. Linke, D. Hunger, J. Reiche, Nature 450, 272 (2007).

[29] K. Zhang, W. Chen, M. Bhattacharya, P. Meystre, Phys. Rev. A 81, 013802 (2010). 
[30] Y.J. Wang, D.Z. Anderson, V.M. Bright, E.A. Cornell, Q. Diot, T. Kishimoto, M. Prentiss, R.A. Saravanan, S.R. Segal, S. Wu, Phys. Rev. Lett. 94, 090405 (2005).

[31] P. Treutlein, D. Hunger, S. Camerer, T.W. Hänsch, J. Reichel, Phys. Rev. Lett. 99, 140403 (2007).

[32] J. Fortágh, C. Zimmermann, Rev. Mod. Phys. 79, 235 (2007).

[33] P.C. Ma, L.L. Yan, J. Zhang, G.B. Chen, X.W. Li, Y.B. Zhan, Laser Phys. Lett. 15015204 (2018). 